\title{
Microbial evolution reshapes soil carbon feedbacks to climate warming
}

\author{
Elsa $\mathrm{Abs}^{1}$, Scott Saleska ${ }^{2}$, and Regis Ferrière ${ }^{3}$ \\ ${ }^{1} \mathrm{UC}$ Irvine \\ ${ }^{2}$ University of Arizona \\ ${ }^{3}$ Ecole Normale Superieure
}

May 5, 2020

\begin{abstract}
Microbial decomposition of soil organic matter is a key component of the global carbon cycle. As Earth's climate changes, the response of microbes and microbial enzymes to rising temperatures will largely determine the soil carbon feedback to atmospheric $\mathrm{CO} 2$. While increasing attention focuses on physiological and ecological mechanisms of microbial responses, the role of evolutionary adaptation remains little studied. To address this gap, we developed an ecosystem-evolutionary model of a soil microbe-enzyme system under warming. Constraining the model with observations from five contrasting sites reveals evolutionary aggravation of soil carbon loss to be the most likely outcome; however, temperature-dependent increases in mortality could cause an evolutionary buffering effect instead. We generally predict a strong latitudinal pattern, from small evolutionary effects at low latitude to large evolutionary effects at high latitudes. Accounting for microbial evolutionary adaptation will likely be critical for improving projections of Earth system responses to climate change.
\end{abstract}

\section{INTRODUCTION}

Microorganisms are key drivers of global biogeochemical cycles (Falkowski et al.2008). In terrestrial ecosystems, soil microbes decompose organic matter, returning carbon to the atmosphere as carbon dioxide $\left(\mathrm{CO}_{2}\right)$ (Waksman \& Starkey 1931). In vitro and in situ experiments suggest that changes in microbial decomposition with warming are an important feedback to climate (Davidson \& Janssens 2006; Singh et al. 2010; Frey et al. 2013). Soil microbial populations may respond to increasing temperature through physiological mechanisms such as individual metabolic adjustment (Bradford et al. 2008; Tucker et al. 2013) and ecological mechanisms such as shifts in population abundance or community composition (Wei et al.2014; Creamer et al. 2015). Given the short generation time, large population sizes and genetic variation of many microbial organisms, evolutionary adaptive responses of microbial populations to warming are also likely (Padfield et al. 2016; Schaum et al. 2017). Indeed, rapid evolutionary adaptation of microbial populations to environmental change, especially temperature gradients (Bennett \& Lenski 2007), is well documented in laboratory systems. However, the functional, ecosystem-level consequences of microbial adaptive evolution remain poorly understood, and how microbial evolutionary adaptation to warming may contribute to carbon-climate feedbacks is unknown (Monroe et al.2018).

Key to microbial decomposition of soil organic matter is the production by microbes of extracellular enzymes (exoenzymes), that diffuse locally in the soil and bind to soil organic matter compounds (Ratledge 1994). Because the fitness cost of exoenzyme production (Harder \& Dijkhuizen 1983) (reduced allocation to growth, Fig. 1a) is paid by individual microbes whereas fitness benefits (larger resource pool) are received by microbial collectives (Velicer 2003), we expect genetic variation in exoenzyme production (Trivedi et al.2016) to be under strong selection (Rainey \& Rainey 2003; Velicer 2003). Our objective is to evaluate 
how exoenzyme production responds to selection under environmental warming, and how the evolutionary response of exoenzyme production impacts decomposition and soil organic carbon stock (SOC). Our focus is on soil bacteria, which typically have a large potential for rapid evolutionary adaptation to environmental change (Koskella \& Vos 2015).

To this end, we develop and analyze a novel ecosystem-evolutionary model, starting with an ecosystem model of microbe-enzyme decomposition first proposed by Allison et al. (2010) (Fig. 1a), and modified to take microbial evolutionary adaptation into account. Amongst ecosystem models of soil microbial decomposition (reviewed in Abs and Ferriere (2020)), Allison et al. 's model is the simplest of the CDMZ type, where $C$ denotes the size of a single pool of SOC, $D$, the size of the dissolved organic carbon (DOC) pool, $M$, the microbial biomass, and $Z$, the size of a single pool of exoenzymes. The focal microbial trait is the fraction of assimilated carbon allocated to exoenzyme production (Sinsabaugh \& Moorhead 1994; Allison 2012; Steinweg et al. 2013), or 'exoenzyme allocation fraction', denoted by $\varphi$. The balance of assimilated carbon, $1-\varphi$, is allocated to microbial growth. The effect of temperature on soil microbial activity is mediated by enzymes kinetics, with exoenzymes driving the decomposition rate, and intra-cellular enzymes involved in resource uptake and microbial biomass synthesis. In general, as temperature increases, Allison et al .'s (2010) ecosystem model predicts a decline in equilibrium SOC due to faster enzyme kinetics, hence a positive feedback to atmospheric $\mathrm{CO}_{2}$ and warming.

To investigate how evolutionary adaptation to warming may affect decomposition, we account for the existence of heritable variation (Alster et al.2016; Trivedi et al. 2016) in the exoenzyme allocation fraction, $\varphi$. We then use evolutionary game theory and adaptive dynamics modeling (Geritzet al. 1998; Brännström et al. 2013) to derive the selection gradient of trait $\varphi$ and compute the microbial evolutionarily stable strategy (ESS), $\varphi^{*}$, at any given temperature. Changing temperature alters the selection gradient, hence $\varphi^{*}$. Knowing how the evolutionarily stable trait value $\varphi^{*}$ changes as temperature increases, we can evaluate how the ecosystem equilibrium changes from both the direct effect of warming on enzyme kinetics, and the indirect effect mediated by microbial evolutionary adaptation to warming (Fig. 1b, c). By comparing the response of the SOC stock that our ecosystem evolutionary model predicts (EVOL response) to the response predicted by the ecosystem $C D M Z$ model in the absence of evolution (ECOS response, assuming $\varphi$ to be a fixed parameter), we can evaluate the contribution of microbial evolutionary adaptation (EVO effect) to the direction and magnitude of the SOC stock response to climate warming (Fig. 1b, c).

To illustrate how EVO effects may vary in real ecosystems, we use available data (Germanet al. 2012) on the decomposition kinetic parameters in five sites of increasing latitude and decreasing mean annual temperature. We evaluate ECOS and EVOL responses for each site, and compare them within and among sites. Our analysis identifies parameters and temperature dependencies that critically influence the strength of evolutionary effects. We discuss how these evolutionary effects relate to previous consideration of 'adaptation' in microbial responses to climate warming (Allisonet al. 2010; Wieder et al. 2013; Allison 2014). We also discuss how, in natural systems, evolutionary adaptation may interact with ecological responses such as species sorting and community shifts (O'Brienet al. 2013; Boon et al. 2014; Strauss 2014). We highlight how our results could inform future empirical work, and conclude with implications for Earth system modeling and forecasting.

\section{METHODS}

\section{Ecosystem model and temperature dependencies}

We use Allison et al.'s (2010) microbe-enzyme model of litter decomposition to describe the ecosystem dynamics of $C, D, M$, and $Z$, given litter input, leaching rates, and soil temperature (equations (1a-d) in Box 1, Fig. 1a and Supplementary Fig. 1). DOC uptaken by individual cells is allocated to exoenzyme production (fraction $\varphi$ ) or microbial biomass, with enzyme production efficiency denoted by $\gamma_{\mathrm{z}}$ and microbial growth efficiency (MGE) denoted by $\gamma_{\mathrm{M}}$.

At the molecular and cellular level, the effect of warming on microbial decomposition is mediated by the temperature sensitivity of intra- and extra-cellular enzymatic activity (Wallensteinet al. 2009; German et 
al. 2012; Burns et al.2013). In the baseline 'kinetics-only' scenario of temperature-dependent decomposition, microbial uptake parameters (maximum uptake rate, half-saturation constant) and exoenzyme kinetics parameters (maximum decomposition rate, half-saturation constant) increase with temperature (Hochachka \& Somero 2002; Davidson \& Janssens 2006) in a logistic manner (equations (2a-d) in Box 1). Two other scenarios have been proposed for the influence of temperature on decomposition. In the microbial mortality scenario, the microbial death rate also increases with temperature (equation (3) in Box 1). This could be due to a higher risk of predation or pathogenic infection at higher temperatures, or faster microbial senescence due to higher protein turnover (Hagerty et al.2014). In the microbial growth efficiency (MGE) scenario, MGE (the fraction of carbon allocated to growth that actually contributes to microbial biomass, as opposed to being released as $\mathrm{CO}_{2}$ via growth respiration) decreases with warming (Allison et al.2010; Wieder et al. 2013) (equation (4) in Box 1), which could be due to higher maintenance costs at higher temperature (Sinsabaugh et al.2013).

The model parameters (microbial life history parameters, thermal dependencies, enzyme parameters, carbon inputs) were constrained by experimental and observational data (Allisonet al. 2010; Allison 2012; German et al. 2012) (see Abs and Ferriere 20 for a review, Supporting Notes 2 for details and Supplementary Table 1). Within these parameter ranges, the ecosystem model outputs are consistent with target empirical values (target $C$ of the order of $100 \mathrm{mg} \mathrm{cm} \mathrm{cm}^{-3}, M$ about $2 \%$ of $C, Z$ about $1 \%$ of $M$, and limiting $D$ close to zero). (Zhanget al. 2014) (2014) provided some more direct validation by successfully fitting a CDMZ model to time series of field measurements of soil respiration from a specific ecosystem (semiarid savannah subject to episodic rainfall pulses). How scenarios of temperature-dependence and parameter values influence the response of equilibrium $C$ to temperature is shown in Supplementary Fig. 4 and commented on in the Supplementary Note 3.

\section{Evolutionary model of exoenzyme production}

Assuming heritable variation in the exoenzyme allocation fraction trait, $\varphi$, we use the framework of adaptive dynamics (Metz et al.1992; Geritz et al. 1998) to predict the strength and direction of selection on trait $\varphi$ and the evolutionarily stable value, $\varphi^{*}$. In this framework, evolution is modeled as a competition process between a 'resident strategy' (wild-type) and alternate strategies (mutants) within a set of feasible phenotypes. In a given environment (e.g. at a given temperature), an evolutionarily stable strategy (ESS) is a phenotype that when resident, no mutant can invade. The adaptive dynamics framework provides the mathematical criteria to identify ESSs and check their attractivity, i.e. that they can be reached by a sequence of small evolutionary steps, each step involving the replacement of a resident phenotype by a mutant phenotype. Here the set of feasible phenotypes is the range $\left(\varphi_{\min }, \varphi_{\max }\right)$ at a given temperature, for which the non-trivial ecosystem equilibrium exists (see Box 1). The derivation of the selection gradient and evolutionarily stable trait value, $\varphi^{*}$, as a function of temperature $T$, is presented in Box 2 .

\section{Ecosystem response (ECOS) and ecosystem evolutionary response (EVOL) to warming}

As temperature rises from $T_{0}$ to $T$, the direction and magnitude of the microbial adaptive response is measured by $\varphi=\varphi^{*}(T)-\varphi^{*}\left(T_{0}\right)$, which depends on the scenario of temperature dependence. The ecosystemevolutionary response (EVOL response) of SOC is given by

(9) EVOL response $=\Delta C_{\text {EVOL }}\left(T_{0}, T\right)=C(T, \varphi *(T))-C\left(T_{0}, \varphi *\left(T_{0}\right)\right)$

where $C(T, \varphi)$ denotes ecosystem equilibrium $C$ at temperature $T$, given enzyme allocation fraction $\varphi$. The EVOL response is to be compared with the response in the absence of evolution (ECOS response):

(10) $\operatorname{ECOS}$ response $=\Delta C_{\operatorname{ECOS}}\left(T_{0}, T\right)=C\left(T_{,} \varphi^{*}\left(T_{0}\right)\right)-C\left(T_{0}, \varphi *\left(T_{0}\right)\right)$

in which the enzyme allocation fraction is fixed at its $T_{0}$-adapted value, $\varphi *\left(T_{0}\right)$ (Fig. 1c).

We measure the magnitude of the evolutionary effect (EVO effect) as the difference between the EVOL response averaged over the temperature range $\left(T_{0}, T\right)$ and the ECOS response averaged over the same temperature range, normalized by the ECOS response: 
(11) EVO effect $=\frac{\left|\int_{T_{0}}^{T} C_{\mathrm{EVOL}}\left(T_{0}, T\right)-\int_{T_{0}}^{T} C_{\mathrm{ECOS}}\left(T_{0}, T\right)\right|}{\left|\int_{T_{0}}^{T} C_{\mathrm{ECOS}}\left(T_{0}, T\right)\right|}$

This evaluation allows us to compare EVO effects across systems that differ in the magnitude of their ECOS response. In all simulations we use $T=T_{0}+\Delta T$ where $\Delta T=5{ }^{\circ} \mathrm{C}$. In general, the ECOS and EVOL responses are monotonic, close-to-linear functions of $T$ over the considered temperature ranges $\left(T_{0}, T_{0}+\Delta T\right)$, which makes all our comparative analyses almost insensitive to our choice of $\Delta T$.

\section{Sensitivity analysis}

We analysed the model sensitivity by varying parameters over two orders of magnitude (as in (Allisonet al. 2010) - except $\gamma_{\mathrm{M}}$ and $\gamma_{\mathrm{z}}$ for which we used the whole range over which the non-trivial ecosystem equilibrium is stable (Supplementary Table 2). To assess the significance of our findings for real ecosystems, we focused on five sites for which empirical data (German et al. 2012) could be used to constrain the model. The five sites contrast strongly in their initial temperature, $T_{0}$, and decomposition kinetics, $v_{\max }^{D}(T)$ and $K_{m}^{D}(T)$ for which we selected the Arrhenius equations (equations (2a-d) in Box 1) that best fit the relations used in (German et al. 2012) (Supplementary Table 3).

\section{RESULTS}

\section{ESS enzyme allocation fraction at given temperature}

The exoenzyme allocation fraction ESS, $\varphi^{*}$, corresponds to a maximum of microbial fitness relative to other trait values, and therefore depends on the parameters defining microbial net growth rate, i.e. MGE $\left(\gamma_{\mathrm{M}}\right)$, maximum uptake rate $\left(v_{\max }^{U}\right)$, mortality $\left(d_{\mathrm{M}}\right)$, and local competitive advantage to exoenzyme producers, or 'competition asymmetry' $\left(c_{0}\right)$ :

$(8) \varphi^{*}(T)=1-\frac{d_{M}}{\gamma_{\mathrm{M}} v_{\max }^{U}}-\frac{1}{c_{0}}$

Note that $c_{0}$, the degree of competition asymmetry, measures the differential availability of enzymatically produced dissolved organic carbon (DOC) to different microbial strains. Competition asymmetry is shaped by diffusion of exoenzymes and DOC, and by microbial mobility, and is thus likely influenced by soil physical properties, such as texture or moisture. For simplicity, we assume that competition asymmetry is independent of temperature.

At a fixed temperature, the model predicts that microbes invest less in exoenzymes when exposed to hostile conditions causing high mortality $\left(d_{\mathrm{M}}\right)$, low $\operatorname{MGE}\left(\gamma_{\mathrm{M}}\right)$, low maximal uptake rate $\left(v_{\max }^{U}\right)$, and/or low competitive advantage to producers $\left(c_{0}\right)$. Conversely, microbes are selected to allocate more resource into exoenzyme production when more favourable growth conditions yield a better 'return on investment' (Schimel \& Weintraub 2003).

\section{Effect of warming on the enzyme allocation fraction ESS}

According to equation (8), the adaptive response of exoenzyme allocation to warming depends on how the MGE, maximum uptake, and mortality vary with temperature. We investigate how parameters influence the direction and sensitivity of the exoenzyme allocation fraction ESS to temperature by calculating the derivative of $\varphi^{*}$ with respect to $T$, for each scenario of temperature dependence (Supplementary Note 4, Supplementary Fig. 5a-d). In the baseline 'kinetics-only' scenario (in which $v_{\max }^{U}$ is the only parameter in equation (8) that depends on temperature), increasing temperature creates more favorable growth conditions through higher resource uptake capacity (higher $v_{\max }^{U}$ ), which selects for higher investment in exoenzyme production (Supplementary Fig. 5a), resulting in lower equilibrium SOC. This is an evolutionary amplification of the positive feedback to warming driven by the ECOS response. Because $\mathrm{d} \varphi * / \mathrm{d} T$ is proportional to both $\exp (1 / T)$ and $1 / T^{2}$, the sensitivity of $\varphi^{*}$ to temperature is strongest across low temperatures (Supplementary Note 4, Supplementary Fig. 5a). The adaptive response is generally greatest (and the amplification of the positive climate feedback strongest) when warming enhances microbial growth potential (by increasing $v_{\max }^{U}$ ) under initially hostile conditions (high $d_{M}$, low $\gamma_{M}$, low $v_{\max }^{U}$ due to low intrinsic uptake rate $v_{0}^{U}$ and/or high activation energy $E_{v}^{U}$, low initial temperature $T_{0}$ ). 
In the temperature-dependent mortality scenario, both mortality and uptake respond exponentially to temperature. Microbes evolve a higher investment in exoenzyme production in response to warming if the change in resource uptake capacity remains greater than the change in mortality rate; otherwise, microbes evolve a lower investment (Supplementary Note 4 and Supplementary Fig. 5b, c). In the MGE-temperature dependent scenario, the response of $\varphi^{*}$ to temperature is proportional to the inverse of an exponential function of temperature (through the uptake rate, $v_{\max }^{U}$ ) times a linear function of temperature (through MGE) (Hagertyet al. 2014). As a consequence, at low initial temperature, the adaptive response of the allocation strategy to warming is mainly driven by the thermal dependence of resource uptake, and microbes adapt by increasing their resource investment in exoenzyme production. For ecosystems that are initially warmer, the microbial evolutionary adaptation to warming (lower allocation to exoenzymes) is mainly driven by the reduction of MGE (Supplementary Note 4 and Supplementary Fig. 5d).

We therefore conclude that microbial evolutionary adaptation to warming generally leads to larger resource allocation to exoenzyme production and a stronger positive carbon feedback to warming, with a greater response in initially colder ecosystems. There are two cases where positive soil C feedbacks to warming (due to higher enzyme kinetic rates) could be attenuated or even reversed: temperature sensitive mortality, or temperature-dependent MGE in an initially warm ecosystem (Supplementary Fig. 5c, d). Next we examine the magnitude of the amplification, attenuation, or reversal of positive carbon feedbacks to warming.

\section{Comparing the ECOS and EVOL responses to warming}

The evolutionary ecosystem response of SOC equilibrium to warming combines the non-evolutionary response of the ecosystem and the evolutionary adaptive response of exoenzyme production (Fig. 1c). In all three scenarios of temperature dependence, the SOC non-evolutionary equilibrium generally decreases as temperature or resource allocation to exoenzymes increases (Fig. 1b, Supplementary Figs. 3 and 4, Supplementary Note 3). The negative effect of temperature results from the SOC equilibrium being mostly sensitive to the maximum decomposition rate $\left(v_{\max }^{D}\right)$ (Supplementary Note 3$)$. As the maximum decomposition rate increases with warming, the SOC equilibrium decreases. The SOC equilibrium is always lower in systems where microbes invest more resources in exoenzymes because, all other parameters being fixed, a larger exoenzyme allocation fraction entails that more exoenzymes are produced per unit time, which leads to more SOC decomposed per unit time (Supplementary Note 1).

We therefore predict that, without evolution, the equilibrium soil carbon stock shrinks as the climate warms, in all scenarios of temperature dependence (Fig. 3). The strongest losses occur in initially cold systems due to the non-linear response of the maximum decomposition rate $\left(v_{\max }^{D}\right)$, hence of the SOC equilibrium, to temperature (Fig. 3, Supplementary Figure 4). We expect microbial evolutionary adaptation to alter the non-evolutionary response according to the scenario, from aggravating soil carbon loss in systems where microbes adapt to warming with higher exoenzyme production, to buffering carbon loss from soils where microbes adaptively respond to warming with a lower exoenzyme allocation fraction (Fig. 1c).

As expected, the simulated ecosystem-evolutionary change in SOC equilibrium in response to warming (Figs. 2, 3) mirrors the evolutionary adaptive response of the exoenzyme allocation fraction to warming in all scenarios of temperature dependence (Supplementary Figs. 5a-d). In the baseline scenario, microbes always evolve a higher enzyme allocation fraction in response to rising temperature; as a consequence, evolutionary adaptation amplifies the non-evolutionary soil carbon loss due to warming (Fig. 3a). The effect of evolution is strongest in ecosystems characterized by conditions that are hostile to microbial growth (low initial temperature, $T_{0}$; high mortality, $d_{M}$; low MGE, $\gamma_{M}$; low maximum uptake rate, $v_{0}^{U}$ ) (Fig. 2, Fig. 3a, Supplementary Fig. 6), where the adaptive response of the exoenzyme allocation fraction is most pronounced (Supplementary Fig. 5a). Strong evolutionary effects are robust to the other model parameters - enzyme parameters (efficiency, production) and environmental parameters (litter input, leaching) (Supplementary Figs. 6 and 7, Supplementary Note 5), which have no influence on the relationship between $\varphi *$ and temperature (equation (8)). The model also predicts stronger evolutionary effects when the differential access to resources between microbial strains is small (low $c_{0}$ ) (equation (8), Fig. 2c, d). Small competition asymmetry selects for microbes allocating little to exoenzymes (low $\varphi^{*}$ ); due to the non-linear response of 
SOC equilibrium to $\varphi$, this causes the SOC equilibrium to be more sensitive to variation in the exoenzyme allocation fraction (Supplementary Note 1, Supplementary Fig. 3).

In the temperature-dependent mortality scenario, the strength of the effect of temperature on mortality is an important determinant of the evolutionary adaptive response to warming. When mortality is moderately sensitive to temperature, the positive response of $\varphi^{*}$ to warming is attenuated. As a result, the evolutionary aggravation of soil carbon loss is less severe (Fig. 3b). When mortality is strongly sensitive to temperature, warming creates more hostile conditions for microbial growth, therefore $\varphi^{*}$ decreases with warming. Evolutionary adaptation then buffers the loss of soil carbon (negative EVO effect, Fig. 3c).

In the temperature-dependent MGE scenario, the direction (positive or negative) of the response of $\varphi *$ to warming is determined by the initial temperature $T_{0}$. The evolutionary effect parallels the response of $\varphi$ *. At low $T_{0}, \varphi^{*}$ increases strongly with temperature, causing an aggravation of soil carbon loss (positive evolutionary effect). In contrast, at high $T_{0}, \varphi^{*}$ decreases strongly with warming, thus opposing the nonevolutionary response (negative evolutionary effect) and promoting carbon sequestration instead (Fig. 3d). At intermediate $T_{0}, \varphi$ * is weakly sensitive to temperature, and the effect of evolutionary adaptation to warming is negligible.

\section{Model predictions using empirical data from five sites}

To illustrate how evolutionary effects may vary in real ecosystems, we used available data (Germanet al. 2012) on the decomposition kinetic parameters from five sites of increasing latitude and decreasing mean annual temperature (Costa Rica, California, West Virginia, Maine, and Alaska, Fig. 4). We evaluated non-evolutionary and evolutionary responses for each site under three levels of competition asymmetry (as quantified by the local competitive advantage to producers, $c_{0}$ ) (Fig. 4a-h). Under our baseline scenario, EVO effects correlate strongly with mean annual temperature, even more so for low competition asymmetry (Fig. 4i). Stronger EVO effects occur in colder sites, as found in the general analysis (Fig. 3a). In contrast, the non-evolutionary response does not correlate with mean annual temperature (Fig. 4a). As a result, a temperate site such as Maine exhibits a weak non-evolutionary response that can be strongly amplified by evolution, whereas the warm Costa Rica site shows a strong non-evolutionary response that is little affected by evolution.

These results are quantitatively attenuated but qualitatively unaffected when microbial mortality increases moderately with temperature (Fig. $4 \mathrm{~b}, \mathrm{f}, \mathrm{j}$ ). With a stronger effect of temperature on microbial mortality, all sites show the evolutionary buffering effect (Fig. 4k) found in the general analysis (Fig. 3c). The intensity of evolutionary buffering is independent of the sites' mean annual temperature, whereas it varies significantly with competition asymmetry (Fig. 4k). Under the temperature-dependent MGE scenario, nonevolutionary and evolutionary responses are reduced in magnitude compared to the baseline scenario (Fig. 4d, h), particularly in cold sites. However, in these sites, EVO effects are enhanced dramatically (Fig. 4l). Thus, in a site as cold as Alaska, a significant evolutionary loss of soil carbon is predicted, whereas the non-evolutionary-driven loss of soil carbon would be negligible (Fig. 4l).

\section{DISCUSSION}

Our general analysis of the ecosystem $C D M Z$ model has shown that the non-evolutionary response of equilibrium SOC to warming always involves soil carbon loss and may only vary in amplitude (Fig. 1). In contrast, the evolutionary adaptive response of microbial exoenzyme allocation, which is shaped by an interaction among the non-linear temperature dependences of microbial traits, can drive negative as well as positive responses of equilibrium SOC to warming. The size of evolutionary effects is most sensitive to MGE, microbial mortality, activation energy of uptake maximal rate, competition asymmetry, initial temperature (Fig. 2), and to the traits' temperature sensitivity (Fig. 3). By specifying the model for five contrasting sites for which exoenzyme kinetics data are available (German et al.2012), we find evolutionary aggravation of soil carbon loss to be the most likely outcome, with a strong latitudinal pattern, from small evolutionary effects at low latitude to large evolutionary effects at high latitudes (Fig. 4). Strong temperature-dependence of microbial mortality, however, would dramatically change the evolutionary pattern, possibly causing an 
attenuation of soil carbon loss or even carbon sequestration in response to warming.

\section{Model predictions and empirical data}

The model results are broadly consistent with empirical work showing that changes in soil C stocks are driven by changes in microbial enzyme activity (Carreiroet al. 2000; Waldrop et al. 2004), and that these changes in activity arise from the multiple effects of temperature on enzyme kinetics, microbial pool size, and microbial allocation to exoenzymes (Steinweg et al. 2013; Malik et al. 2019). Empirical data suggest that natural values of enzyme allocation fraction are low (Schimel \& Weintraub 2003; Burns et al. 2013) and fall in the range for which our model predicts large evolutionary responses of decomposition to warming. Measured mass-specific potential enzyme activity, used as a proxy for allocation to exoenzymes, was shown to generally increase with warming, a pattern also predicted by our evolutionary model (Steinweg et al.2013). In particular, Steinweg et al . found that the rise in enzyme allocation was greatest for moderate warming and less pronounced for strong warming, which matches our model predictions under the scenario of temperature-dependent MGE.

A large body of empirical observations and controlled experiments at various time and spatial scales highlights a general response of soil respiration and soil $\mathrm{C}$ stocks to warming, involving an ephemeral increase of respiration, no significant change in SOC, and a decrease in microbial biomass. Only the temperaturedependent mortality scenario with evolution could match these patterns. It has been argued, however, that most experiments remain insufficient to rule out model predictions that depart from these patterns (Sulman et al.2018). Furthermore, the mechanism (physiological, ecological and/or evolutionary) by which exoenzyme allocation and soil respiration vary in these experiments is unknown (see Supplementary Note 6 for further discussion of ecosystem modeling predictions of soil $\mathrm{C}$ responses to warming). Next we discuss the potential interplay of evolutionary adaptation with physiological acclimation (phenotypic plasticity within individuals) and ecological community assembly (shifts in species composition in whole communities) in shaping soil decomposition responses to warming.

\section{Evolutionary adaptation vs. physiological acclimation}

Acclimation responses, whereby individuals' physiological mechanisms buffer the kinetics effect of warming and maintain homeostasis in key life-history traits such as growth and maintenance, are sometimes referred to as 'adaptation' (e.g. of microbial carbon use efficiency in response to warming ) (Allisonet al. 2010; Wieder et al. 2013; Allison 2014). However, this is phenotypic plasticity at the individual level, rather than evolutionary adaptation at the population level. In our model, constant mortality (in the baseline and temperature-dependent MGE scenarios) and constant MGE (in the baseline and temperature-dependent mortality scenarios) can be interpreted as manifestations of microbial acclimation (Allison et al.2010; Hagerty et al. 2014). Our results show that such plasticity, whereby individual cells buffer growth efficiency and/or mortality against temperature variation, does not necessarily impede or overwhelm evolutionary adaptation to climate warming. It is precisely under the assumptions that MGE and mortality remain constant with respect to temperature that the strongest adaptive change in exoenzyme allocation is predicted (see Supplementary Note 7 for further discussion of implications regarding 'adaptation' of microbial carbon-use efficiency).

The exoenzyme allocation fraction itself might be plastic. Indeed, Steinweg et al. (2013) interpreted the positive effect of temperature on allocation to enzyme production as a cell-level response to larger nutrient needs driven by higher maintenance costs. In our model, warming causes larger rates of nutrient uptakes (higher $v_{\max }^{U}$ ) and this can lead to an adaptive increase of the enzyme allocation fraction (equation (8)). From the general evolutionary theory of phenotypic plasticity (Scheiner 1993), we expect the evolutionary optimal reaction norm of enzyme allocation fraction to follow the same pattern, i.e. $\varphi$ increasing with temperature, provided that the cost of plasticity is not too high and does not alter the selection gradient too markedly. This warrants future investigation.

An interesting way of testing the role of evolutionary adaptation vs. plasticity would be to monitor the effect of warming on experimentally evolving bacterial communities at different levels of medium diffusivity or porosity (Rebolleda-Gómez \& Travisano 2018). Our model predicts that the diffusion of resources (DOC), that is 
likely dependent on physical properties of the soil medium, has a strong influence on the adaptive evolution of microbial exoenzyme production in response to warming, but not on the ecosystem (non-evolutionary) response driven by enzyme kinetics and physiological plasticity. Thus, comparing population-scale decomposition and respiration across treatments that factorially cross temperature and medium physical properties may help disentangle the effects of adaptation vs. plasticity.

\section{Evolutionary adaptation and ecological community responses}

In natural soils exposed to climate change, microbial evolutionary adaptation is expected to interplay with ecological responses such as species sorting or community shifts in species or functional group abundances (O'Brienet al. 2013; Boon et al. 2014; Strauss 2014). Indeed, there is growing experimental evidence for soil bacterial communities shifting, with functional consequences on decomposition, in response to climate (Glassman et al. 2018). This raises the challenge of assessing the relative importance of evolutionary and ecological mechanisms in the response of microbial decomposition to warming. Available empirical evidence suggest that both could be significant. For example, recent experiments onPseudomonas bacteria showed that local evolutionary adaptation was as important as community composition in shaping the community response to elevated temperature over the course of a two-month experiment (Gómez et al. 2016).

Our model could be extended to integrate evolutionary adaptation and ecological shifts in response to warming. To represent the functional diversity of a microbial community exploiting a diversity of substrates, different SOC pools could be included (Wang et al. 2013), alongside specifying different types of enzymes and different microbial functional groups that produce them (Allison 2012). In our model parameterization, microbial functional groups would potentially differ in traits such as MGE $\left(\gamma_{M}\right)$, enzyme cost $\left(\gamma_{Z}\right)$ and uptake rate $\left(v_{\max }^{U}\right)$ (Allison 2012), and assuming heritable variation, the evolution of these traits would drive the adaptive response of the microbial functional community to environmental change. In an evolution experiment using Neurospora discreta as a model system to assess adaptation of soil fungi to warming (Romero-Olivareset al. 2015), Allison et al. (2018) did find evidence for evolutionary responses in MGE and uptake rates. By letting multiple traits evolve, our model extended to multiple substrates could be used first to generate an evolutionarily stable community at a given temperature (following Sauterey et al .'s (Sauterey et al.2015) approach for including evolutionary dynamics in models of ocean planktonic communities), and then evaluate both ecological and evolutionary responses to warming.

\section{Scaling up}

In spite of an increasing effort to document and understand the ecosystem impact of microbial physiological and ecological responses to climate warming (Allisonet al. 2010; Treseder et al. 2012; Wieder et al.2013), no Earth system model that seeks to represent the role of living organisms in climate feedbacks has yet included evolutionary mechanisms of adaptation. This model is a critical first step. To predict geographic variation in evolutionary responses and effects across large geographic scales, our results highlights the need for more empirical data on variation in ecosystem (competition) traits, especially how the competitive advantage to exoenzyme producers varies with soil physical properties, and in microbial physiological traits, especially microbial mortality (see Supplementary Note 8 for further discussion of the effect of microbial traits' temperature dependence on ecosystem-evolutionary responses). On the modeling side, three steps will be needed: accounting for soil carbon stabilization on a timescale longer than respiration (Tang \& Riley 2014); for vegetation types, the associated diversity of organic substrates in litter, and corresponding diversity of microbial decomposers (Allison 2012); and for the interaction of biogeochemical cycles and associated stoichiometric constraints (Harte \& Kinzig 1993; Sinsabaugh \& Moorhead 1994; Schimel \& Weintraub 2003; Allison 2012; Kaiser et al. 2015). As demonstrated by successful Earth-scale modeling of phytoplankton abundance and distribution in the global ocean (Followset al. 2007), future models that take these steps will trade off some of their added structural complexity with the model 'self-parameterization' driven by the process of adaptive trait evolution itself. As this research program unfolds, we expect projections of future climate and carbon cycle feedbacks, and their uncertainty, to be significantly impacted by microbial evolutionary adaptation, from local to global scales. 


\section{ACKNOWLEDGEMENTS}

We thank Rachel Gallery, Pierre-Henri Gouyon, Moira Hough, Hélène Leman, Laura Meredith, and Mitch Pavao-Zuckerman for discussion. E.A. was supported by fellowships from Ecole Doctorale Frontières du Vivant and MemoLife Laboratory of Excellence (PIA-10-LBX-54). S.R.S. was supported by a grant from the Genomic Science Program of the United States Department of Energy (DOE) (DE-SC0016440) and the University of Arizona's Agnese Nelms Haury Program in Environment and Social Justice. R.F. acknowledges support from FACE Partner University Fund, CNRS Mission pour l'Interdisciplinarité, PSL University (IRIS OCAV and PSL-University of Arizona Mobility Program) and a grant from the Dimensions of Biodiversity program of the United States National Science Foundation (DEB-1831493).

\section{REFERENCES}

Allison, S.D. (2012). A trait-based approach for modelling microbial litter decomposition.Ecol. Lett., 15, 1058-1070.

Allison, S.D. (2014). Modeling adaptation of carbon use efficiency in microbial communities. Front. Microbiol., 5,571 .

Allison, S.D., Wallenstein, M.D. \& Bradford, M.A. (2010). Soil-carbon response to warming dependent on microbial physiology. Nat. Geosci., 3, 336 .

Alster, C.J., Baas, P., Wallenstein, M.D., Johnson, N.G. \& von Fischer, J.C. (2016). Temperature Sensitivity as a Microbial Trait Using Parameters from Macromolecular Rate Theory. Front. Microbiol., 7, 1821.

Bennett, A.F. \& Lenski, R.E. (2007). An experimental test of evolutionary trade-offs during temperature adaptation. Proc. Natl. Acad. Sci. U. S. A., 104 Suppl 1, 8649-8654.

Boon, E., Meehan, C.J., Whidden, C., Wong, D.H.-J., Langille, M.G.I. \& Beiko, R.G. (2014). Interactions in the microbiome: communities of organisms and communities of genes. FEMS Microbiol. Rev., 38, 90-118.

Bradford, M.A., Davies, C.A., Frey, S.D., Maddox, T.R., Melillo, J.M., Mohan, J.E., et al.(2008). Thermal adaptation of soil microbial respiration to elevated temperature. Ecol. Lett., 11, 1316-1327.

Brännström, A., Johansson, J. \& von Festenberg, N. (2013). The Hitchhiker's Guide to Adaptive Dynamics. Games, An Introduction to Population Genetics Theory, 4, 304-328.

Burns, R.G., DeForest, J.L., Marxsen, J., Sinsabaugh, R.L., Stromberger, M.E., Wallenstein, M.D.,et al. (2013). Soil enzymes in a changing environment: Current knowledge and future directions. Soil Biol. Biochem., 58, 216-234.

Carreiro, M.M., Sinsabaugh, R.L., Repert, D.A. \& Parkhurst, D.F. (2000). MICROBIAL ENZYME SHIFTS EXPLAIN LITTER DECAY RESPONSES TO SIMULATED NITROGEN DEPOSITION.Ecology, 81, $2359-2365$.

Creamer, C.A., de Menezes, A.B., Krull, E.S., Sanderman, J., Newton-Walters, R. \& Farrell, M. (2015). Microbial community structure mediates response of soil C decomposition to litter addition and warming. Soil Biol. Biochem., 80, 175-188.

Davidson, E.A. \& Janssens, I.A. (2006). Temperature sensitivity of soil carbon decomposition and feedbacks to climate change. Nature, 440, 165-173.

Driscoll, W.W. \& Pepper, J.W. (2010). Theory for the evolution of diffusible external goods.Evolution, 64, $2682-2687$.

Falkowski, P.G., Fenchel, T. \& Delong, E.F. (2008). The microbial engines that drive Earth's biogeochemical cycles. Science, 320, 1034-1039.

Follows, M.J., Dutkiewicz, S., Grant, S. \& Chisholm, S.W. (2007). Emergent biogeography of microbial communities in a model ocean. Science, 315, 1843-1846. 
Frey, S.D., Lee, J., Melillo, J.M. \& Six, J. (2013). The temperature response of soil microbial efficiency and its feedback to climate. Nat. Clim. Chang., 3, 395.

Geritz, S.A.H., Kisdi, E., Mesze`NA, G. \& Metz, J.A.J. (1998). Evolutionarily singular strategies and the adaptive growth and branching of the evolutionary tree.Evol. Ecol., 12, 35-57.

German, D.P., Marcelo, K.R.B., Stone, M.M. \& Allison, S.D. (2012). The Michaelis-Menten kinetics of soil extracellular enzymes in response to temperature: a cross-latitudinal study. Glob. Chang. Biol., 18, 14681479 .

Glassman, S.I., Weihe, C., Li, J., Albright, M.B.N., Looby, C.I., Martiny, A.C., et al.(2018). Decomposition responses to climate depend on microbial community composition. Proc. Natl. Acad. Sci. U. S. A., 115, 11994-11999.

Gómez, P., Paterson, S., De Meester, L., Liu, X., Lenzi, L., Sharma, M.D., et al. (2016). Local adaptation of a bacterium is as important as its presence in structuring a natural microbial community. Nat. Commun., 7,12453 .

Hagerty, S.B., van Groenigen, K.J., Allison, S.D., Hungate, B.A., Schwartz, E., Koch, G.W., et al. (2014). Accelerated microbial turnover but constant growth efficiency with warming in soil. Nat. Clim. Chang., 4, 903.

Harder, W. \& Dijkhuizen, L. (1983). Physiological responses to nutrient limitation. Annu. Rev. Microbiol., $37,1-23$.

Harte, J. \& Kinzig, A.P. (1993). Mutualism and competition between plants and decomposers: implications for nutrient allocation in ecosystems. Am. Nat., 141, 829-846.

Hochachka, P.W. \& Somero, G.N. (2002). Biochemical Adaptation: Mechanism and Process in Physiological Evolution. Oxford University Press.

Kaiser, C., Franklin, O., Richter, A. \& Dieckmann, U. (2015). Social dynamics within decomposer communities lead to nitrogen retention and organic matter build-up in soils. Nat. Commun., 6, 8960.

Koskella, B. \& Vos, M. (2015). Adaptation in Natural Microbial Populations. Annu. Rev. Ecol. Evol. Syst., $46,503-522$.

Li, J., Wang, G., Allison, S.D., Mayes, M.A. \& Luo, Y. (2014). Soil carbon sensitivity to temperature and carbon use efficiency compared across microbial-ecosystem models of varying complexity.Biogeochemistry, $119,67-84$.

Malik, A.A., Puissant, J., Goodall, T., Allison, S.D. \& Griffiths, R.I. (2019). Soil microbial communities with greater investment in resource acquisition have lower growth yield. Soil Biol. Biochem., 132, 36-39.

Metz, J.A.J., Nisbet, R.M. \& Geritz, S.A.H. (1992). How should we define "fitness" for general ecological scenarios? Trends Ecol. Evol., 7, 198-202.

Monroe, J.G., Markman, D.W., Beck, W.S., Felton, A.J., Vahsen, M.L. \& Pressler, Y. (2018). Ecoevolutionary Dynamics of Carbon Cycling in the Anthropocene.Trends Ecol. Evol., 33, 213-225.

O'Brien, S., Hodgson, D.J. \& Buckling, A. (2013). The interplay between microevolution and community structure in microbial populations. Curr. Opin. Biotechnol., 24, 821-825.

Padfield, D., Yvon-Durocher, G., Buckling, A., Jennings, S. \& Yvon-Durocher, G. (2016). Rapid evolution of metabolic traits explains thermal adaptation in phytoplankton. Ecol. Lett., 19, 133-142.

Rainey, P.B. \& Rainey, K. (2003). Evolution of cooperation and conflict in experimental bacterial populations. Nature, 425, 72-74. 
Ratledge, C. (1994). Biodegradation of oils, fats and fatty acids. In: Biochemistry of microbial degradation (ed. Ratledge, C.). Springer Netherlands, Dordrecht, pp. 89-141.

Rebolleda-Gómez, M. \& Travisano, M. (2018). The Cost of Being Big: Local Competition, Importance of Dispersal, and Experimental Evolution of Reversal to Unicellularity. Am. Nat., 192, 731-744.

Romero-Olivares, A.L., Taylor, J.W. \& Treseder, K.K. (2015). Neurospora discreta as a model to assess adaptation of soil fungi to warming. BMC Evolutionary Biology.

Sauterey, B., Ward, B.A., Follows, M.J., Bowler, C. \& Claessen, D. (2015). When everything is not everywhere but species evolve: an alternative method to model adaptive properties of marine ecosystems. J. Plankton Res., 37, 28-47.

Schaum, C.-E., Barton, S., Bestion, E., Buckling, A., Garcia-Carreras, B., Lopez, P., et al.(2017). Adaptation of phytoplankton to a decade of experimental warming linked to increased photosynthesis. Nat Ecol Evol, 1, 94.

Scheiner, S.M. (1993). Genetics and evolution of phenotypic plasticity. Annu. Rev. Ecol. Syst., 24, 35-68.

Schimel, J.P. \& Weintraub, M.N. (2003). The implications of exoenzyme activity on microbial carbon and nitrogen limitation in soil: a theoretical model. Soil Biol. Biochem., 35, 549-563.

Singh, B.K., Bardgett, R.D., Smith, P. \& Reay, D.S. (2010). Microorganisms and climate change: terrestrial feedbacks and mitigation options. Nat. Rev. Microbiol., 8, 779-790.

Sinsabaugh, R.L., Manzoni, S., Moorhead, D.L. \& Richter, A. (2013). Carbon use efficiency of microbial communities: stoichiometry, methodology and modelling.Ecol. Lett., 16, 930-939.

Sinsabaugh, R.L. \& Moorhead, D.L. (1994). Resource allocation to extracellular enzyme production: A model for nitrogen and phosphorus control of litter decomposition.Soil Biol. Biochem., 26, 1305-1311.

Steinweg, J.M., Dukes, J.S., Paul, E.A. \& Wallenstein, M.D. (2013). Microbial responses to multi-factor climate change: effects on soil enzymes. Front. Microbiol., 4, 146.

Stone, M.M., Weiss, M.S., Goodale, C.L., Adams, M.B., Fernandez, I.J., German, D.P., et al.(2012). Temperature sensitivity of soil enzyme kinetics under N-fertilization in two temperate forests. Glob. Chang. Biol., $18,1173-1184$.

Strauss, S.Y. (2014). Ecological and evolutionary responses in complex communities: implications for invasions and eco-evolutionary feedbacks. Oikos, 123, 257-266.

Sulman, B.N., Moore, J.A.M., Abramoff, R., Averill, C., Kivlin, S., Georgiou, K., et al.(2018). Multiple models and experiments underscore large uncertainty in soil carbon dynamics. Biogeochemistry, 141, 109123.

Tang, J. \& Riley, W.J. (2014). Weaker soil carbon-climate feedbacks resulting from microbial and abiotic interactions. Nat. Clim. Chang., 5, 56.

Treseder, K.K., Balser, T.C., Bradford, M.A., Brodie, E.L., Dubinsky, E.A., Eviner, V.T., et al. (2012). Integrating microbial ecology into ecosystem models: challenges and priorities. Biogeochemistry, 109, 7-18.

Trivedi, P., Delgado-Baquerizo, M., Trivedi, C., Hu, H., Anderson, I.C., Jeffries, T.C., et al. (2016). Microbial regulation of the soil carbon cycle: evidence from gene-enzyme relationships. ISME J., 10, 2593-2604.

Tucker, C.L., Bell, J., Pendall, E. \& Ogle, K. (2013). Does declining carbon-use efficiency explain thermal acclimation of soil respiration with warming? Glob. Chang. Biol., 19, 252-263.

Velicer, G.J. (2003). Social strife in the microbial world. Trends Microbiol., 11, 330-337.

Waksman, S.A. \& Starkey, R.L. (1931). The soil and the microbe. John Wiley And Sons; New York. 
Waldrop, M.P., Zak, D.R., Sinsabaugh, R.L., Gallo, M. \& Lauber, C. (2004). NITROGEN DEPOSITION MODIFIES SOIL CARBON STORAGE THROUGH CHANGES IN MICROBIAL ENZYMATIC ACTIVITY. Ecol. Appl., 14, 1172-1177.

Wallenstein, M.D., Mcmahon, S.K. \& Schimel, J.P. (2009). Seasonal variation in enzyme activities and temperature sensitivities in Arctic tundra soils. Glob. Chang. Biol., 15, 1631-1639.

Wang, G., Post, W.M. \& Mayes, M.A. (2013). Development of microbial-enzyme-mediated decomposition model parameters through steady-state and dynamic analyses. Ecol. Appl., 23, 255-272.

Wei, H., Guenet, B., Vicca, S., Nunan, N., AbdElgawad, H., Pouteau, V., et al. (2014). Thermal acclimation of organic matter decomposition in an artificial forest soil is related to shifts in microbial community structure.Soil Biol. Biochem., 71, 1-12.

Wieder, W.R., Bonan, G.B. \& Allison, S.D. (2013). Global soil carbon projections are improved by modelling microbial processes. Nat. Clim. Chang., 3, 909.

Zhang, X., Niu, G.-Y., Elshall, A.S., Ye, M., Barron-Gafford, G.A. \& Pavao-Zuckerman, M. (2014). Assessing five evolving microbial enzyme models against field measurements from a semiarid savannah-What are the mechanisms of soil respiration pulses? Geophys. Res. Lett., 41, 6428-6434.

\section{Figure Captions}

Figure 1. Effect of temperature and enzyme allocation fraction on SOC ecosystem equilibrium. a , Structure of the microbial-enzyme ecosystem model (see Methods for details): SOC stock is the balance of plant input, $I$, and loss by exoenzyme-mediated degradation to DOC $(D)$, which in turn is allocated between (fraction $\varphi$ ) production of exoenzymes $(Z)$ and (fraction 1- $\varphi$ ) growth of microbial biomass $(M)$. b , Effect of temperature and exoenzyme allocation fraction, $\varphi$, on SOC equilibrium, $C$, in the baseline scenario of temperature dependence. c , Response of SOC ecosystem equilibrium, $C$, to a $5^{\circ} \mathrm{C}$ increase in temperature (from $20{ }^{\circ} \mathrm{C}$ to $25{ }^{\circ} \mathrm{C}$ ) as a function of exoenzyme allocation fraction, $\varphi$. Parameters are set to their default values (Supplementary Table 1), except $I=510^{-3}, v_{0}^{U}=10^{5}, E_{v}^{U}=38, c_{0}=1.17$.

Figure 2. Effect of microbial evolutionary adaptation on the SOC equilibrium response to + $5{ }^{\circ} \mathbf{C}$ warming (EVO effect). Temperature influences enzyme kinetics only (baseline scenario of temperature dependence). a , Influence of microbial biomass production efficiency, $\gamma_{\mathrm{M}}$, and microbial mortality rate, $d_{\mathrm{M}}$. b , Influence of microbial resource acquisition traits $v_{0}^{U}$ and $E_{v}^{U}$. c-d , Influence of competition asymmetry, $c_{0}$, and initial temperature, $T_{0}$. In all figures, constant parameters are set to their default values (Supplementary Table 1 ) and $I$ is set to $510^{-3}$. Points A1 and B1 indicate the default parameter values. Point A2 (respectively B2) exemplifies values of $\gamma_{\mathrm{M}}$ and $d_{\mathrm{M}}$ (resp. $v_{0}^{U}$ and $E_{v}^{U}$ ) for which the EVO effect is strong. Panel c (resp. d ) shows the influence of $c_{0}$ and $T_{0}$ on the EVO effect at A2 (resp. B2).

Figure 3. Ecosystem (ECOS) and ecosystem-evolutionary (EVOL) responses of SOC equilibrium to warming (up to $+5{ }^{\circ} \mathrm{C}$ ) for three scenarios of temperature dependence. Ecosystem and ecosystem-evolutionary changes in SOC equilibrium $C$ given by equation (10) (without evolution, dashed curves) and equation (9) (with evolution, plain curves) are plotted as a function of the increase in temperature. Blue curves, initial temperature $T_{0}=5^{\circ} \mathrm{C}$. Black curves, $T_{0}=T_{\text {ref }}=20^{\circ} \mathrm{C}$. Red curves, $T_{0}=30^{\circ} \mathrm{C}$. Insets, Direction and magnitude of EVO effect (\%), from $-150 \%$ to $+150 \%$, color code indicates $T_{0}$ as before. a , Baseline scenario of temperature dependence (enzyme kinetics only).b , Temperature-dependent microbial mortality, with $E_{\mathrm{dM}}=25<E_{v}^{U}$. c , Temperature- dependent microbial mortality with $E \mathrm{dM}=55$ $>E_{v}^{U}$.d , Temperature-dependent MGE, with $m=0.014$. Parameters values correspond to point B2 in Fig. 2 $\left(I=510^{-3}, v_{0}^{U}=10^{5}, E_{v}^{U}=38, c_{0}=1.17\right.$ ); other parameters are set to their default values (Supplementary Table 1).

Figure 4. Ecosystem (ECOS) and ecosystem-evolutionary (EVOL) responses of SOC equilibrium to $+5{ }^{\circ} \mathrm{C}$ warming predicted for five sites. a-d, ECOS response. e-h, EVOL response. i-l, EVO effect given by equation (11). AK: Alaska, boreal forest, $T_{0}=0.1^{\circ} \mathrm{C}$. ME: Maine, temperate forest, $T_{0}=$ 
$5^{\circ} \mathrm{C}$. WV: West Virginia, temperate forest, $T_{0}=9^{\circ} \mathrm{C}$. CA: California, temperate grassland, $T_{0}=17^{\circ} \mathrm{C}$. CR: Costa Rica, tropical rain forest, $T_{0}=26^{\circ} \mathrm{C}$. First row $(\mathbf{a}, \mathbf{e}, \mathbf{i})$ : baseline kinetics-only scenario of temperature dependence. Second row $(\mathbf{b}, \mathbf{f}, \mathbf{j})$ : temperature-dependent microbial mortality scenario with $E \mathrm{dM}=25<$ $E_{v}^{U}$. Third row $(c, g, k)$ : temperature- dependent microbial mortality scenario with $E \mathrm{dM}=55>E_{v}^{U}$. Fourth row $(\mathbf{d}, \mathbf{h}, \mathbf{l})$ : temperature-dependent MGE scenario $(m=-0.014)$. The influence of competition asymmetry, $c_{0}$, is shown (low: $c_{0}=1.17$, intermediate: $c_{0}=1.34$, high: $c_{0}=1.5$ ). For clarity, vertical axis for ECOS and EVOL responses are truncated at $-65 \mathrm{mg} \mathrm{C} \mathrm{cm}^{-3}$. Actual values for AK with $c{ }_{0}=1.17$ are ECOS response $=-170 \mathrm{mg} \mathrm{C} \mathrm{cm}{ }^{-3}$ and EVOL response $=-556 \mathrm{mg} \mathrm{C} \mathrm{cm}^{-3}$; actual value for $\mathrm{WV}$ with $c_{0}=$ 1.17 is EVOL response $=-92.8 \mathrm{mg} \mathrm{C} \mathrm{cm}^{-3}$. Parameter values correspond to point B2 in Fig. $2\left(I=510^{-3}\right.$, $v_{0}^{U}=10^{5}, E_{v}^{U}=38, c_{0}=1.17$ ); other parameters are set to their default values (Supplementary Table 1).
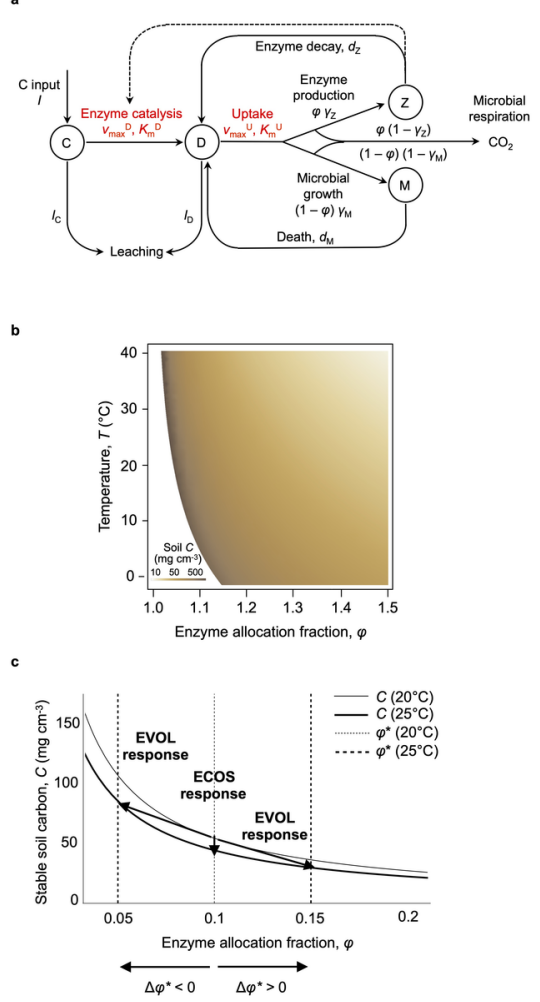

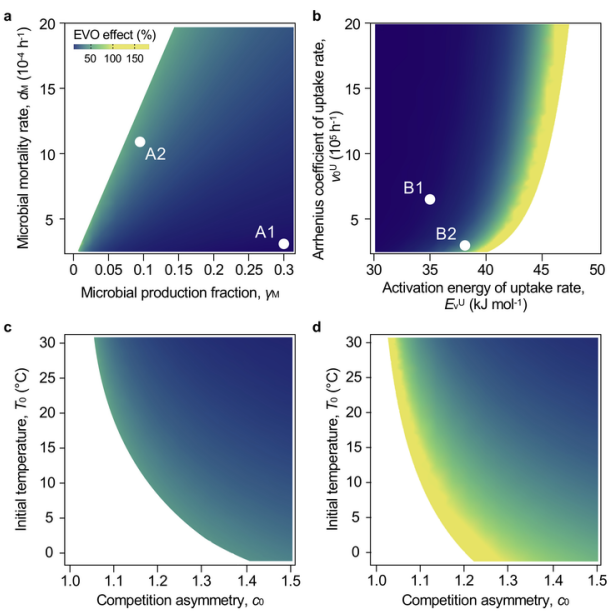

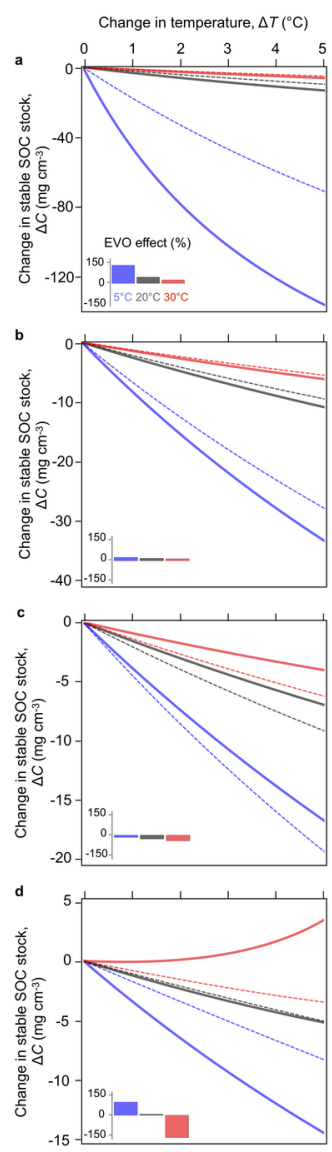


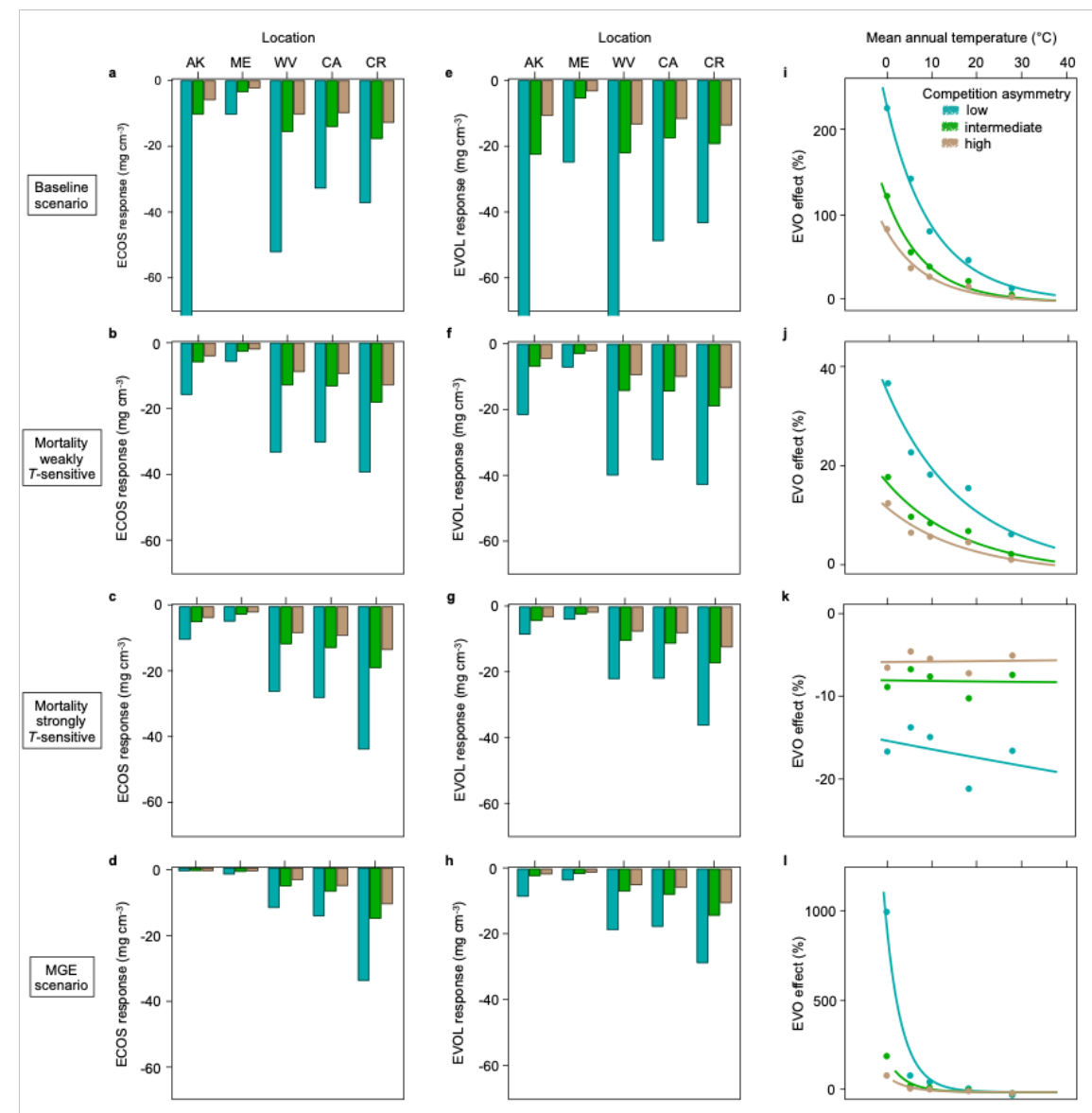

\section{Box 1: Ecosystem $C D M Z$ model and temperature dependencies.}

Based on (Allison et al. 2010) (Fig. 1a, Supplementary Fig. 1), the ecosystem model has four state variables measured in unit mass of carbon: soil (non decomposed) organic carbon (SOC), $C$; soil decomposed soluble organic carbon (DOC), $D$; microbial biomass, $M$; and exoenzyme concentration, $Z$. Exoenzyme production drives the decomposition process of $C$ into $D$, which is the only source of carbon for microbes. The model accounts for microbial production and death, exoenzyme decay, recycling of dead microbes and degraded exoenzymes, $C$ input from plant litter, and leaching of $C$ and $D$.

Model equations. State variables $C, D, M, Z$ obey equations (1a-d):

(1a) $\frac{\mathrm{dC}}{\mathrm{dt}}=I-\frac{v_{\max }^{D} C}{K_{m}^{D}+C} Z-e_{C} C$

(1b) $\frac{\mathrm{dD}}{\mathrm{dt}}=\frac{v_{\max }^{D} C}{K_{m}^{D}+C} Z+d_{M} M+d_{Z} Z-\frac{v_{\max }^{U} D}{K_{m}^{U}+D} M-e_{D} D$

(1c) $\frac{\mathrm{dM}}{\mathrm{dt}}=(1-\varphi) \gamma_{M} \frac{v_{\max }^{U} D}{K_{m}^{U}+D} M-d_{M} M$

(1d) $\frac{\mathrm{dZ}}{\mathrm{dt}}=\varphi \gamma_{Z} \frac{v_{\max }^{U} D}{K_{m}^{U}+D} M+d_{Z} Z$

In equation (1a), decomposition follows from Michaelis-Menten kinetics of $Z$ binding substrate $C$; there is a constant input, $I$, of soil organic (non decomposed) carbon from aboveground litter, and a loss due to leaching at constant rate $e_{\mathrm{C}}$. In equation (1b), $D$ is produced by decomposition and the recycling of dead microbial biomass and inactive enzymes; $D$ is consumed by microbial uptake, and lost by leaching at 
constant rate $e_{\mathrm{D}}$. In equation (1c), growth of microbial biomass $M$ is driven by the rate of $D$ uptake (a Monod function of $D$ ) times the fraction of uptaken $D$ turned into biomass, $(1-\varphi) \gamma_{\mathrm{M}}$, minus microbial mortality at constant rate $d_{\mathrm{M}}$. In equation (1d), enzyme variation is driven by the rate of $D$ uptake times the fraction allocated to enzyme production, $\varphi$, and production efficiency, $\gamma \mathrm{z}$, minus enzyme deactivation at constant rate, $d \mathrm{z}$.

Ecosystem equilibria. The ecosystem model (equations (1a-d)) possesses either one globally stable equilibrium, or three equilibria (one of which is always unstable) (Supplementary Note 1, Supplementary Fig. 2). There are thresholds $\varphi_{\min }$ and $\varphi_{\max }$ such that the single globally stable equilibrium exists for $\varphi<\varphi \min$ or $\varphi>\varphi$ max and is given by $C=I / e_{C}, D=0, M=0, Z=0$. Thus, at this equilibrium, the microbial population is extinct and no decomposition occurs. For $\varphi \min <\varphi<\varphi \max$, the microbial population can either go extinct (then the system stabilizes at the same equilibrium as before) or persists at or around a non-trivial equilibrium, which can be solved for analytically (Supplementary equation (1)). Note that $\varphi_{\text {min }}$ and $\varphi$ max depend on all microbial and model parameters (Supplementary Note 1, Supplementary Fig. 3).

Effect of temperature on model parameters. Decomposition is predicted to respond to warming (Davidson \& Janssens 2006) due to the temperature sensitivity of enzymatic activity (Wallensteinet al. 2009; German et al. 2012; Stone et al.2012). Microbial assimilation may also vary with temperature if the microbial membrane proteins involved in nutrient uptake are sensitive to warming. Following (Allisonet al. 2010), we assume that exoenzyme kinetics parameters (maximum decomposition rate $v_{\max }^{D}$ and half-saturation constant $K_{m}^{D}$ ) and microbial uptake parameters (maximum uptake rate $v_{\max }^{U}$ and half-saturation constant $K_{m}^{U}$ ) follow Arrhenius relations with temperature. This defines our baseline 'kinetics-only' scenario of temperaturedependent decomposition:

$$
\begin{aligned}
& \text { (2a) } v_{\max }^{D}=v_{0}^{D} e^{-\frac{E_{v}^{D}}{R(T+273)}} \\
& (2 \mathrm{~b}) K_{m}^{D}=K_{0}^{D} e^{-\frac{E_{K}^{D}}{R(T+273)}} \\
& \text { (2c) } v_{\max }^{U}=v_{0}^{U} e^{-\frac{E_{v}^{U}}{R(T+273)}} \\
& \text { (2d) } K_{m}^{U}=K_{0}^{U} e^{-\frac{E_{K}^{U}}{R(T+273)}}
\end{aligned}
$$

where $T$ is temperature in Celsius, $R$ is the ideal gas constant, and the $E$ parameters denote the corresponding activation energies.

We consider two additional scenarios for the influence of temperature on decomposition. In the temperaturedependent microbial mortality scenario (Hagerty et al.2014), the microbial death rate $d{ }_{\mathrm{M}}$ depends on temperature according to

$$
(3) d_{M}(T)=d_{M 0} e^{-\frac{E_{\mathrm{dM}}}{R(T+273)}}
$$

as in (Hagerty et al.2014).

In the temperature-dependent microbial growth efficiency (MGE) scenario, the MGE decreases with temperature (Allisonet al. 2010; Wieder et al. 2013; Hagerty et al.2014), which is modeled by making the microbial growth efficiency $\gamma$ м vary linearly with temperature (Allisonet al. 2010; German et al. 2012; Wang et al. 2013; Li et al. 2014):

(4) $\gamma_{M}(T)=\gamma_{M, \text { ref }}-m\left(T-T_{\text {ref }}\right)$

with $T$ ref $=20^{\circ} \mathrm{C}$.

\section{Box 2: Adaptive dynamics analysis.}

Interaction between resident and mutant strains. To model the competition effect of a resident phenotype, $\varphi$ res, on the population growth of a mutant phenotype, $\varphi$ mut, we extend the ecosystem model written for a single type (equation (1c) in Box 1). To account for the local nature of the interaction between rare mutant 
and common resident cells, we introduce a function (hereafter denoted by $c$ ) of the difference between $\varphi$ res and $\varphi$ mut to measure how local decomposition by mutant and resident cells differ from 'mean field' (average) decomposition by resident cells. Thus, for given $C, D, Z$, the growth of the mutant population is governed by

(5) $\frac{d M_{\mathrm{mut}}}{\mathrm{dt}}=(1-\varphi) \gamma_{M} \frac{v_{\mathrm{max}}^{U}\left(1+c\left(\varphi_{\mathrm{mut}}-\varphi_{\mathrm{res}}\right)\right) D_{\mathrm{res}}}{K_{m}^{U}+\left(1+c\left(\varphi_{\mathrm{mut}}-\varphi_{\mathrm{res}}\right)\right) D_{\mathrm{res}}} M_{\mathrm{mut}}-d_{M} M_{\mathrm{mut}}$

where $D$ res is the equilibrium $D$ predicted by the ecosystem model for the resident phenotype $\varphi$ res. Here function $c$ satisfies $c(0)=0, c(z)>0$ if $z>0$ and $c(z)<0$ if $z<0$.

The underlying assumption is that each microbe has access to DOC partly as a public good and partly as a private good (Driscoll \& Pepper 2010). The public good part results from the diffusion of exoenzymes. The private good part results from local decomposition at the microscopic scale of cells and exoenzymes that they produce themselves. A mutant cell that invests more (resp. less) in exoenzyme has access to more (less) DOC than the average resident cell because the cell's private good is greater (smaller) whereas all cells share the same public good. In a spatially implicit model like ours, diffusion is not directly modeled, but its effect on the accessibility of DOC to a mutant strain can be phenomenologically accounted for by a parameterization that puts mutant cells at a competitive advantage for DOC if the mutant phenotype invests more in exoenzyme production than the resident phenotype, or at a competitive disadvantage if the mutant phenotype invests less. This parameterization is achieved with the function $c$ in equation (5), where $c<1$ when $\varphi$ mut $<\varphi$ res and $c>1$ when $\varphi$ mut $>\varphi$ res. This phenomenological approach is consistent with the mathematical construction and numerical analysis of a spatially explicit model of resident-mutant local interaction that accounts for soil diffusion, which will be reported elsewhere.

Invasion fitness and selection gradient. Mutant fitness $\left(\varphi_{\mathrm{mut}}, \varphi_{\mathrm{res}}\right)$ is given by the mutant population growth rate per unit biomass:

(6) $s\left(\varphi_{\text {mut }}, \varphi_{\text {res }}\right)=(1-\varphi) \gamma_{M} \frac{v_{\text {max }}^{U}\left(1+c\left(\varphi_{\text {mut }}-\varphi_{\text {res }}\right)\right) D_{\text {res }}}{K_{m}^{U}+\left(1+c\left(\varphi_{\text {mut }}-\varphi_{\text {res }}\right)\right) D_{\text {res }}}-d_{M}$

The selection gradient then obtains by taking the first order derivative of the invasion fitness with respect to the mutant trait:

$(7) \nabla s(\varphi)=\frac{d_{M}}{1-\varphi}\left(\left(1-\varphi-\frac{d_{M}}{v_{\max }^{U} \gamma_{M}}\right) c_{0}-1\right)$

where $c_{0}=c^{\prime}(0)$ measures the local competitive advantage to stronger exoenzyme producers, which we call 'competition asymmetry'. Note that by definition of function $c$, we always have $c_{0}>0$. Variation in $c_{0}$ may be caused by different soil diffusion properties, due to e.g. physical texture or moisture.

Evolutionary singularity. Trait values that nullify the selection gradient are called 'evolutionary singularities'. An evolutionary singularity can be attractive or repelling, and invadable or non-invadable. Evolutionary singularities that are attractive and non-invadable represent potential end-points of evolutionary adaptation. Evolutionary singularities that are attractive and invadable can lead to evolutionary branching (Geritz et al.1998).

In a given environment (fixed parameters, constant temperature) there is at most one evolutionary singularity given by defining $\varphi^{*}$ as the value of $\varphi$ that makes $\nabla s(\varphi)=0$ in equation $(7)$ :

$(8) \varphi^{*}=1-\frac{d_{M}}{v_{\max }^{U} \gamma_{M}}-\frac{1}{c_{0}}$.

Existence of $\varphi^{*}>0$ requires $\frac{d_{M}}{v_{\max }^{U} \gamma_{M}}<1$ and $c_{0}>\frac{1}{\left(1-\frac{d_{M}}{v_{\max }^{U} \gamma_{M}}\right)}$. Thus, the (cooperative) trait $\varphi$ can evolve above zero only if the local competition advantage to stronger enzyme producers is large enough. The condition for $\varphi^{*}$ to be evolutionarily stable is $c^{\prime \prime}(0)<2 c_{0}^{2}$ and no other condition than existence is required for $\varphi^{*}$ to be always convergent. Here we assume that function $c$ is such that $\varphi^{*}$ is evolutionarily stable and attractive. 
Equation (8) shows that more cooperation (larger $\varphi^{*}$ ) should evolve in microbial populations with lower mortality, greater nutrient uptake, and/or higher MGE. When comparing microbial populations with similar life-history traits $\gamma_{\mathrm{M}}, v_{\max }^{U}$ and $d_{\mathrm{M}}$, stronger competitive advantage to exoenzyme producers (i.e. higher $c$ 0 ) selects for larger $\varphi^{*}$. 